\title{
Synthesis and characterization of novel flavonoid derivatives via sequential phosphorylation of Quercetin
}

Francis J. Osonga $^{\mathrm{a}}$, Joab O. Onyango ${ }^{\mathrm{b}}$, Samuel K. Mwilu ${ }^{\mathrm{a}}$, Naomih M.Noah ${ }^{\mathrm{a}}$, Jürgen Schulte ${ }^{\mathrm{b}}$, Ming An $^{\mathrm{b}}$, Omowunmi A. Sadik ${ }^{\mathrm{a}} *$

\footnotetext{
${ }^{a}$ Department of Chemistry, Center for Research in Advanced Sensing Technologies \& Environmental Sustainability (CREATES), State University of New York at Binghamton, P.O Box 6000 Binghamton, NY, 13902 USA.

${ }^{b}$ Department of Chemistry, State University of New York at Binghamton, P.O Box 6000 Binghamton, NY, 13902 USA
}

\section{ARTICLE INFO}

\section{Article history:}

Received

Received in revised form

Accepted

Available online

\section{Keywords:}

Keyword_1

Keyword_2

Keyword_3

Keyword_4

Keyword_5

\section{ABSTRACT}

Flavonoids are naturally-occurring polyphenolics that have been implicated in a wide range of biological activities. The major obstacles to flavonoid applications are the poor solubility in common solvents. Phosphorylation of flavonoids yields a new class of flavonoid derivatives which are very soluble in aqueous solutions and hence have the potential to be used in biological studies. We hereby report the sequential phosphorylation of Quercetin leading to the synthesis of Quercetin pentaphosphate (QPP), Apigenin triphosphate (ATRP), 5, 4'-Quercetin Diphosphate (5, 4'-QDP) and 4'- Quercetin monophosphate (4'-QP) with solubility of $848 \mathrm{mg} / \mathrm{mL}, 367$ $\mathrm{mg} / \mathrm{mL}, 315 \mu \mathrm{g} / \mathrm{mL}$ and $106 \mu \mathrm{g} / \mathrm{mL}$ respectively. The synthesis of 4'- QP, 5, 4'- QDP, QPP and ATRP) was successful with $85 \%, 60.5 \%, 56 \%$ and $99 \%$ yield respectively These compounds have been characterized using ${ }^{1} \mathrm{H}-\mathrm{NMR},{ }^{13} \mathrm{C}-\mathrm{NMR},{ }^{31} \mathrm{P}-\mathrm{NMR}$.

Flavonoids constitute a large family of plant-derived polyphenolic compounds that are found in fruits, vegetables, spices, wines and juices ${ }^{1}$. Examples of flavonoids include quercetin, fisetin, luteolin, apeginin, myricetin and many others. Previous studies have shown that these compounds exhibit an array of biological effects that are beneficial to humans, including antiviral, antioxidative, anti-inflammatory and anticarcinogenic.

The solubility of these polyphenolic compounds can be increased through the modification of the chemical structure, which may improve oral bioavailability ${ }^{2}$. As a result, considerable efforts have been directed towards the development of novel, highly soluble conjugates having clinical profiles that are similar or even superior to those exhibited by the parent molecules in vitro. These include synthetic esters, acyl

* Corresponding author. Tel.: +16077774132; fax: +1-607-777-4478

; e-mail: osadik@binghamton.edu 
(methyl, ethyl, propyl etc), phenylisocyanated quercetin derivatives, as well as the quercetin conjugates (methylated, sulfated, glucuronidated, glutathionated, and the isomers of mono, di- and mixed conjugates) ${ }^{3 \text {, }}$ ${ }^{4}$. Recently, some phosphorylated quercetin glycosides were synthesized and reported to exhibit improved solubility with comparable activity towards cultured human malignant cells ${ }^{5}$. Several attempts have also been made to phosphorylate quercetin and some of its derivatives ${ }^{6-10}$ so as to improve its pharmaceutical or physiological activities but none of these studies have been successful in completely phosphorylating the parent molecule. Studies have shifted to the protection of the hydroxyl groups of polyphenols. ${ }^{3,6,7,11 .}$

Currently there is significant interest in the modification of flavonoids to improve the solubility and stability under intracellular conditions by carrying out selective protection of hydroxyl groups. ${ }^{3,} 11$ Recently, Quercetin pivaloxymethyl (POM) analogues were synthesized and it was revealed that the 7POM was more soluble in PBS (pH 7.4) buffer than 3-POM. Although 3-POM was found to be stable, the study revealed that it could not penetrate the cells. ${ }^{11}$ Recently Yingling et al (2013) investigated in vitro studies of the synthesized ester phosphorylated flavonoids as inhibitors of pancreatic cholesterol esterase and acetylcholinesterase. ${ }^{3}$ The study revealed that the modified flavonoids showed remarkable potency against those targets than the corresponding parent flavonoids and the position of the phosphate group has significant effect on the inhibitory activities with the 7-phosphate most potent.

In the last decade, our group has been actively involved in the study of polyphenols, ranging from their ability to affect the viability and proliferation of cancerous cells and to understand their mode of action ${ }^{12-13}$. Until recently, the degradation products of quercetin in biological-like conditions has not been systematically isolated, characterized nor the degradation mechanisms successfully elucidated ${ }^{12}$. We reported the first complete isolation and structural elucidation of 18 intermediates following the electrochemical oxidation of quercetin ${ }^{14}$. Extensive studies by Zhou et al. have been critical in providing deeper insights into underlying mechanisms of quercetin oxidation in different systems ${ }^{14}$.The nature of interaction of quercetin with nucleotides has also been reported ${ }^{13}$.

It is desirable to selectively add only one phosphate group via sequential phosphorylation and selective protection in order to investigate the biological implication of each phosphate on the flavonoid. Hence the 
goal of this work is to (i) synthesize a new class of phosphorylated Quercetin derivatives via sequential phosphorylation (ii) account for the position and nature of the global phosphorylated Quercetin (QCR) and demonstrate the sequential phosphorylated synthesis of Quercetin diphosphate (QDP), Quercetin monophosphate (QP) and Apigenin triphosphate (ATRP).

In this phosphorylation work, we adopted Silverberg's method ${ }^{15}$ which employs in situ generation of chlorodibenzylphosphate as the electrophiles starting from a stable dibenzyl phosphite. ${ }^{11,16-20}$ It is simple, rapid and clean compared to other methods Scheme 1. The elegance of the reaction rests in the instability of the in situ generated chlorodibenzylphosphite as well as the nucleophilic phenoxide generated in situ.
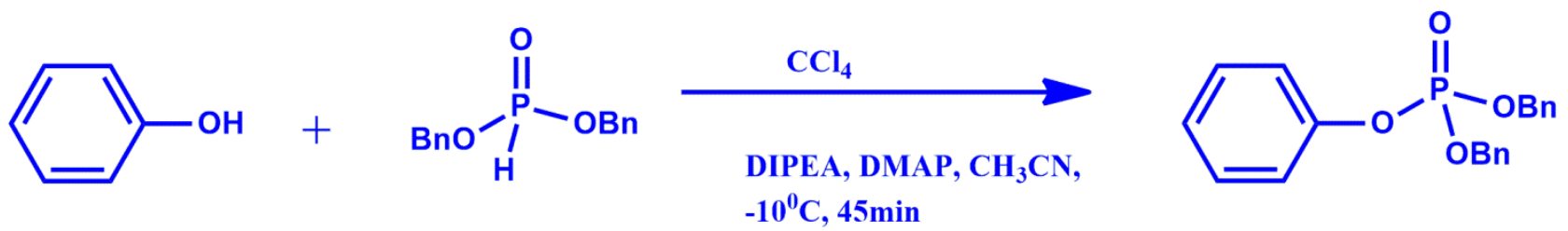

Scheme 1: General Phosphorylation using Dibenzyl Phosphite, N, N-Diisopropylethylamine (DIPEA), Dimethylaminopyridine (DMAP) and carbon tetrachloride $\left(\mathrm{CCl}_{4}\right)$

This reaction was used to install dibenzyl phosphate moieties on free hydroxyls groups on Quercetin.

Organophosphates form a principal means in the multi-billion dollar agricultural, household and pest control industry among other commercial uses. ${ }^{21}$ Even though phosphate exists in many biological systems from small molecules as phosphoenol pyruvate (PEP) to fairly large conjugates such as adenosine triphosphate (ATP), not many drugs of organophosphorus nature have advanced to clinical trials. Our encouraging bioactivity result on polyphosphorylated flavonoids is supported by the existence of fosfomycin - an organophosphonate broad spectrum antibiotic marketed as monurol. ${ }^{22}$ With advances in nucleic acid chemistry, newer phosphorylation methods have been developed including tetrazole-catalyzed method involving phosphoramidite compounds. ${ }^{23,24}$ Furthermore, Lewis acid catalysis have been used in the cases where the donor is not sufficiently nucleophilic. ${ }^{25}$ Recently, development of electrochemical synthesis of organophosphorus was reported. ${ }^{26}$ Phosphorylation of phenols with these methods gives moderate yields and may not be great if applied to late stage synthetic products. The matter is complicated if other functionalities exist in the molecule that need to be handled carefully with appropriate protectiondeprotection manipulations. 

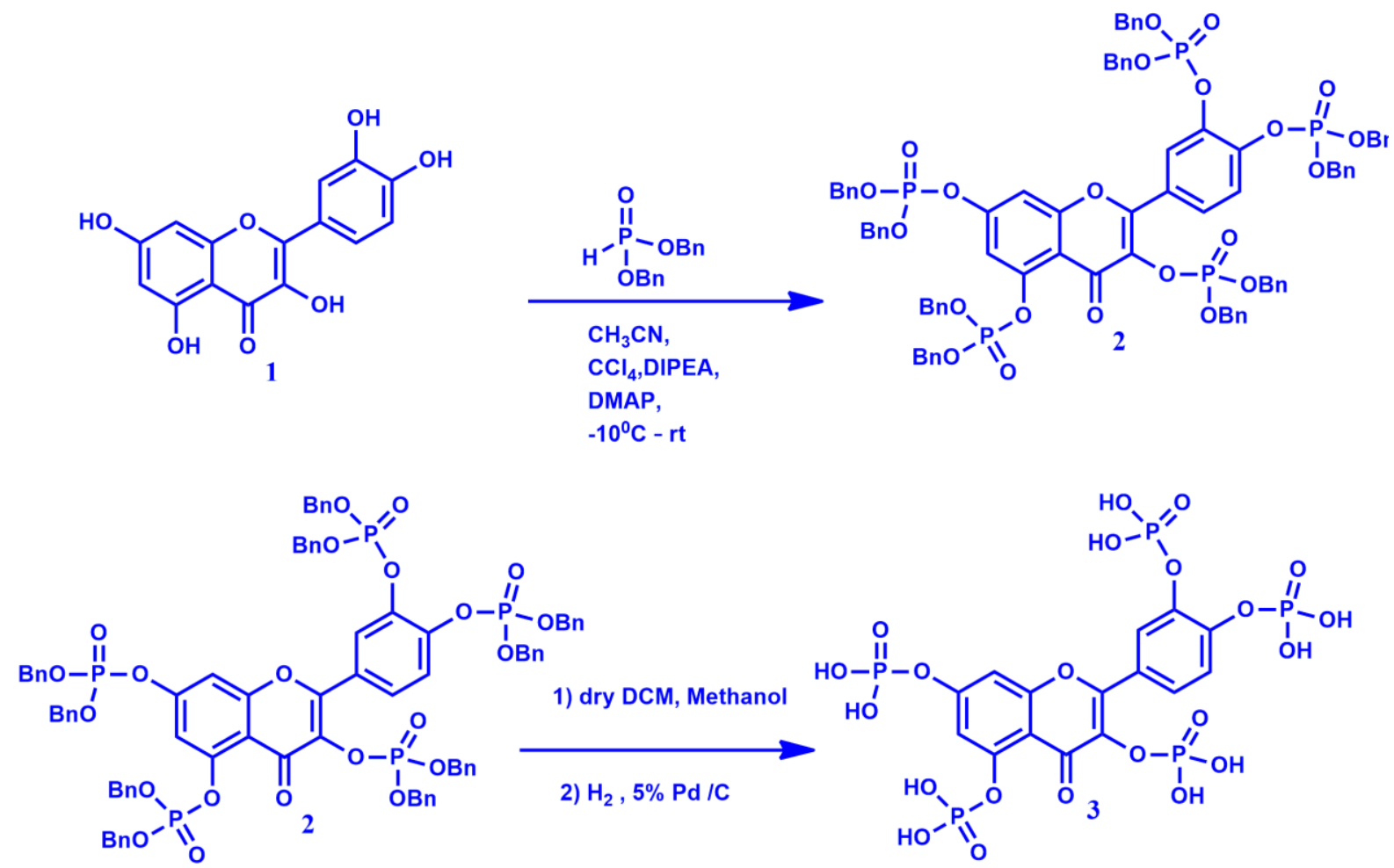

Scheme 2: Synthesis of Quercetin Pentaphosphate Intermediate (QPPI) (2) and Quercetin Pentaphosphate (QPP) (3)

Compounds 2, $35,6,7,8,9$ and 10 were synthesized following the procedure highlighted in the Supplementary section. Scheme 2 shows the synthesis of compounds $\mathbf{2 ,} \mathbf{3}$ and in this case we used DMSO$\mathrm{d}_{6}$ as NMR solvent as opposed to $\mathrm{CDCl}_{3}$. The latter has $\delta$ ppm resonating at 7.26ppm in the ${ }^{1} \mathrm{H}$ NMR which coincides with the chemical shifts of the aromatic protons in the molecule reducing the ability to integrate the area under the peaks corresponding to the proton number. Hence, use of DMSO- $\mathrm{d}_{6}$ with chemical shift at $2.50 \mathrm{ppm}$ in the ${ }^{1} \mathrm{H}$ NMR allows us to account for all the protons in the molecule. We were therefore able to account for 20 methylene protons and 55 aromatic protons for QPPI (2) by integration corresponding to the claimed structure (Figure S1C).

The synthesis of 4'- Quercetin monophosphate (QP), 5, 4'-Quercetin Diphosphate (QDP), Quercetin pentaphosphate (QPP) 3 and Apigenin triphosphate (ATRP) was successful with 85\%, 60.5 \%, 56\% and 99\% yield respectively. However, the synthesis of 5- monophosphate intermediate (QPI) failed. The NMR of compounds 8 and 9 were in agreement with the literature. ${ }^{1}$ The NMR data (Figures 2-3 and Figure S1 S3) of the compounds $\mathbf{2}, \mathbf{3}, \mathbf{7}, \mathbf{8}, \mathbf{9}$ and $\mathbf{1 0}$ were in agreement with the structures. From the synthesis of 
compounds 5 and 6, (Scheme 3) it can be deduced that the benzylation of Quercetin with 3.5 equivalent of benzyl bromide and $\mathrm{K}_{2} \mathrm{CO}_{3}$ results mainly in the production of compound 5 (59.84\%) and $\mathbf{6}(19.65 \%)$ which is in agreement with ratio obtained in literature. ${ }^{11}$

The ${ }^{1} \mathrm{H}$ and ${ }^{13} \mathrm{C}$ NMR results in the experimental section depicts similar trend with slight variation caused by $\mathrm{CDCl}_{3}$ used by the literature ${ }^{11}$ source as opposed to DMSO- $\mathrm{d}_{6}$ which we used. The choice of DMSO- $\mathrm{d}_{6}$ is justified because $\mathrm{CDCl}_{3}$ resonates around $7 \mathrm{ppm}$ which is the region for benzylic protons. From literature studies it is a fact that the 5 position of Quercetin or any other flavonoids cannot be benzylated easily in the conditions stated in the synthesis since the $5-\mathrm{OH}$ is less acidic than the rest of the phenolic moieties, thus providing justification about why it is not possible to install phosphate at position 5 of the parent Quercetin. This fact is attributed to an acid weakening effect of an intramolecular H-bond between the 5-hydroxyl group and the 4-keto group. ${ }^{27,}{ }^{28}$ Selective protection of the Quercetin catechol ring is a prerequisite for effective sequential phosphorylation of Quercetin. In recent years synthesis of 4', 5, 7 monophosphate analogues have been attempted by employing the protection strategy of the O-dihydroxyphenyl group using dichlorophenylmethane. 9,11 The strategy being adopted in this study depends heavily on the difference in reactivity sites of the phenolic groups and the selective protection of the catechol group. ${ }^{3,11}$

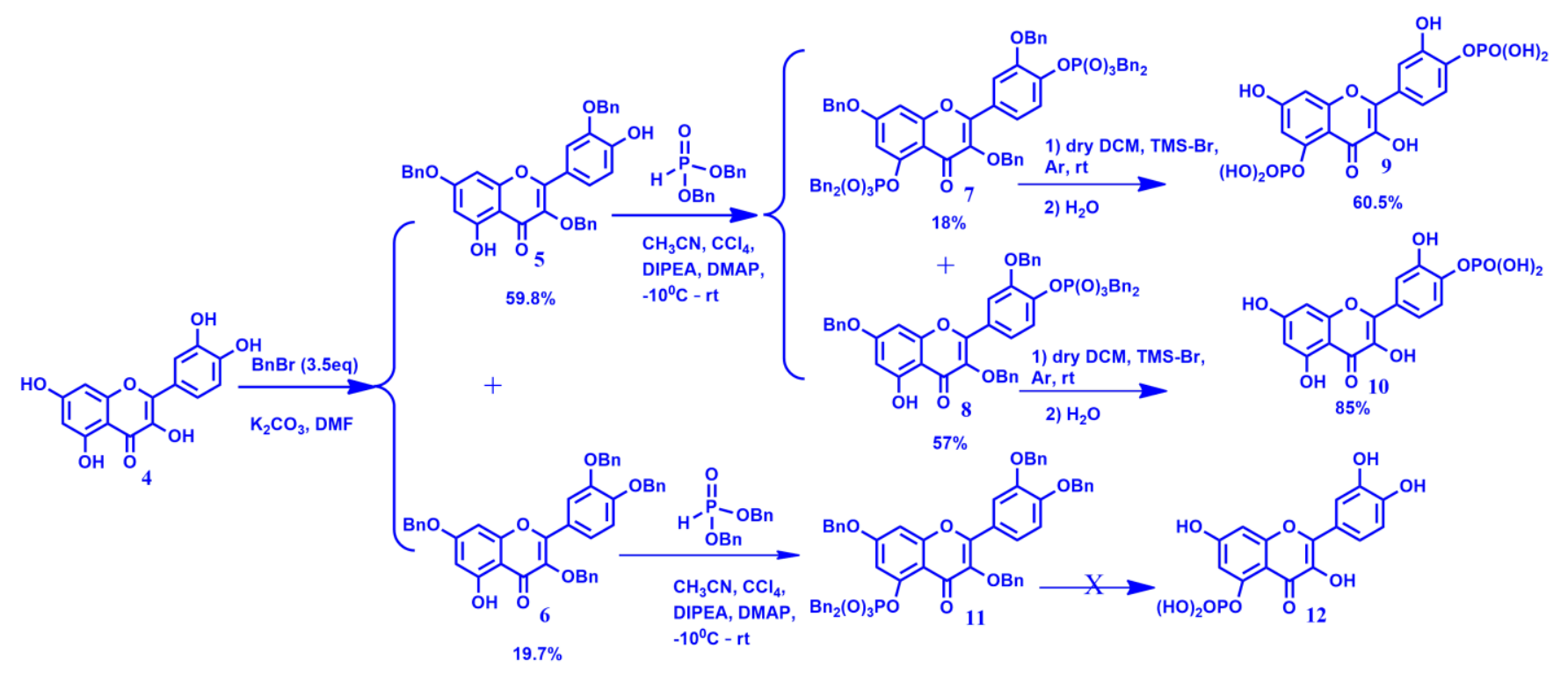

Scheme 3: Sequential synthesis of Quercetin phosphate derivatives

With the $\mathrm{OH}$ group at positions 5 and 4', we anticipated that using Silverberg treatment ${ }^{15}$ (1 equivalent of dibenzyl phospite) will preferentially avail 4'-Quercetin phosphate Intermediate (4'-QPI compound 8) as 
the only phosphate intermediate. Though we isolated 4'-QPI as yellow oily compound we also isolated a diphosphate compound as yellow oily compound as a minor product from the reaction which turned out to be 5, 4'-Quercetin Diphosphate Intermediate (5, 4'-QDPI compound 7). The proton NMR for 5, 4'- QDPI and 4'-QPI confirmed the identity of the compounds. From the NMR data the number of methylene $\left(\mathrm{CH}_{2}\right)$ protons was found to be 14 , the benzylic protons was 35 and the aromatic proton being 5 , totaling to 40 protons for 5, 4'- QDPI. On the other hand the numbers of protons for methylene, benzylic and aromatic protons were found to be 12,30 and 5 respectively yielding a total of 35 protons.<smiles>O=c1cc(-c2ccc(O)cc2)oc2cc(OP(=O)(O)OCc3ccccc3)cc(OP(=O)(OCc3ccccc3)OCc3ccccc3)c12</smiles>

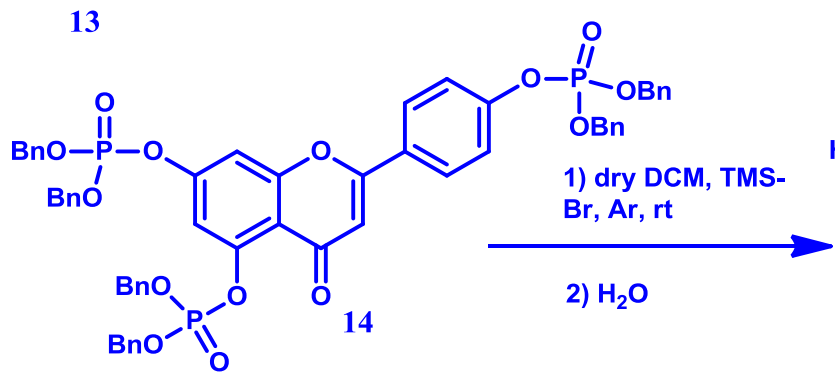<smiles>O=c1cc(-c2ccc(OP(=O)(O)O)cc2)oc2cc(OP(=O)(O)O)cc(OP(=O)(O)O)c12</smiles>

Scheme 4: Synthesis of Apigenin Triphosphate Intermediate (14) and Apigenin Triphosphate (15)

${ }^{31} \mathrm{P}$ NMR as a structural tool for identification in the field of organophosphorus chemistry is strongly influenced by the ${ }^{31} \mathrm{P}$ chemical shifts to the structural environment. ${ }^{29},{ }^{30}$ Chemical shifts provide valuable information about the nearest neighbours of the $\mathrm{P}$ atom in the molecule. ${ }^{29}$ Since organophosphorus became useful in many industrial, agricultural and household products, several studies have been undertaken in structural elucidation techniques. However, no unified theory of factors affecting ${ }^{31} \mathrm{P}$ chemical shifts and coupling constants has been reached. ${ }^{29-31}$ A theoretical quantum-mechanical calculation proposes that electronegativity differences in the P-X bond, changes in the $\pi$ - electron overlap, and changes in the bond angle play significant role. ${ }^{31,32}$ Recently, Pedrosa et al reported a correlation of ab-initio calculation of chemical shifts with experimental data of substituted aryl dialkyl phosphates and concluded that $\delta{ }^{31} \mathrm{P}$ NMR chemical shifts were mainly governed by symmetry of electron cloud on the phosphorus atom and that is 
directly related to the electronegativity of the substituent. Their finding implicates the effect of backbonding from phosphoryl oxygen to phosphorus atom ${ }^{33}$, and was further confirmed by Koo's density functional theory (DFT) studies. ${ }^{29}$ Flavonoid modified with phosphate groups and their intermediates are similar to aryl phosphates studied by Pedrosa. They are tetracoordinate phosphorus compound and the benzyl protected intermediate will differ from its acid counterparts by the enhanced d-orbital occupancy in the former ${ }^{33}$, a character that will enhance back-bonding from the phosphoryl oxygen atom to phosphorus. ${ }^{30}$ A review by Wigfield found that ${ }^{31} \mathrm{P}$ NMR signal of dibenzyl phosphate resonates at $+1.1 \mathrm{ppm}$ (Chemical shifts with respect to $\left.85 \% \mathrm{H}_{3} \mathrm{PO}_{4}\right)^{34}$, however, recent experimental reports ${ }^{19,35-36}$, and theoretical predictions ${ }^{33}$ have indicated that the numbers could be more downfield. Our ${ }^{31} \mathrm{P}$ chemical shifts for QPPI (2), ATRPI (14), 4'-QPI (10) and 5, 4'-QDPI (9) are as shown in Figures 2, 3 and S1-S3. Pedrosa reported that the chemical shift of phosphorus in the aryl phosphate resonates around $-7 \mathrm{ppm}$ and vary depending on the magnitude of electronegativity of substituents on the aryl ring. ${ }^{33}$ The ${ }^{31} \mathrm{P}$ with proton decoupling and without proton decoupling (Figure 3, 4 and S1) confirmed that phosphate was successfully installed giving 2 peaks and 1 peak for 5, 4'-QDPI and 4'-QPI respectively.

Characteristic of benzyl protected phosphate is the signature quintet splitting pattern in the ${ }^{31} \mathrm{P}$ peaks as shown in Figure 1. The presence of $4 \mathrm{H}$ three bonds away, splits the $\mathrm{P}$ nucleus signal into 5 peaks with considerable $\mathrm{J}$ coupling constants a factor likely to be attributed to magnetic properties of phosphorus. ${ }^{29}$

We speculate that for 5, 4'- QDPI the -7.27 ppm peak may be at 4' unlike the 5 which could be at -5.86 regions. The differences in the phosphates on catecholic hydroxyls 4' and 5 in $\delta$ ppm could be due to p $\pi$ - $d \pi$ bonding interaction between aryl oxygen and phosphorus; and this effect results from the substitution at para and meta positions of the phenyl ring. ${ }^{29,} 31$ Position 4' lies directly para to the bond projecting to ring $\mathrm{C}$ of the flavone structure and therefore decreases the $\mathrm{d}$ orbital occupation on phosphorus and shifts the ${ }^{31} \mathrm{P}$ signal to lower fields ${ }^{29,30}$. 


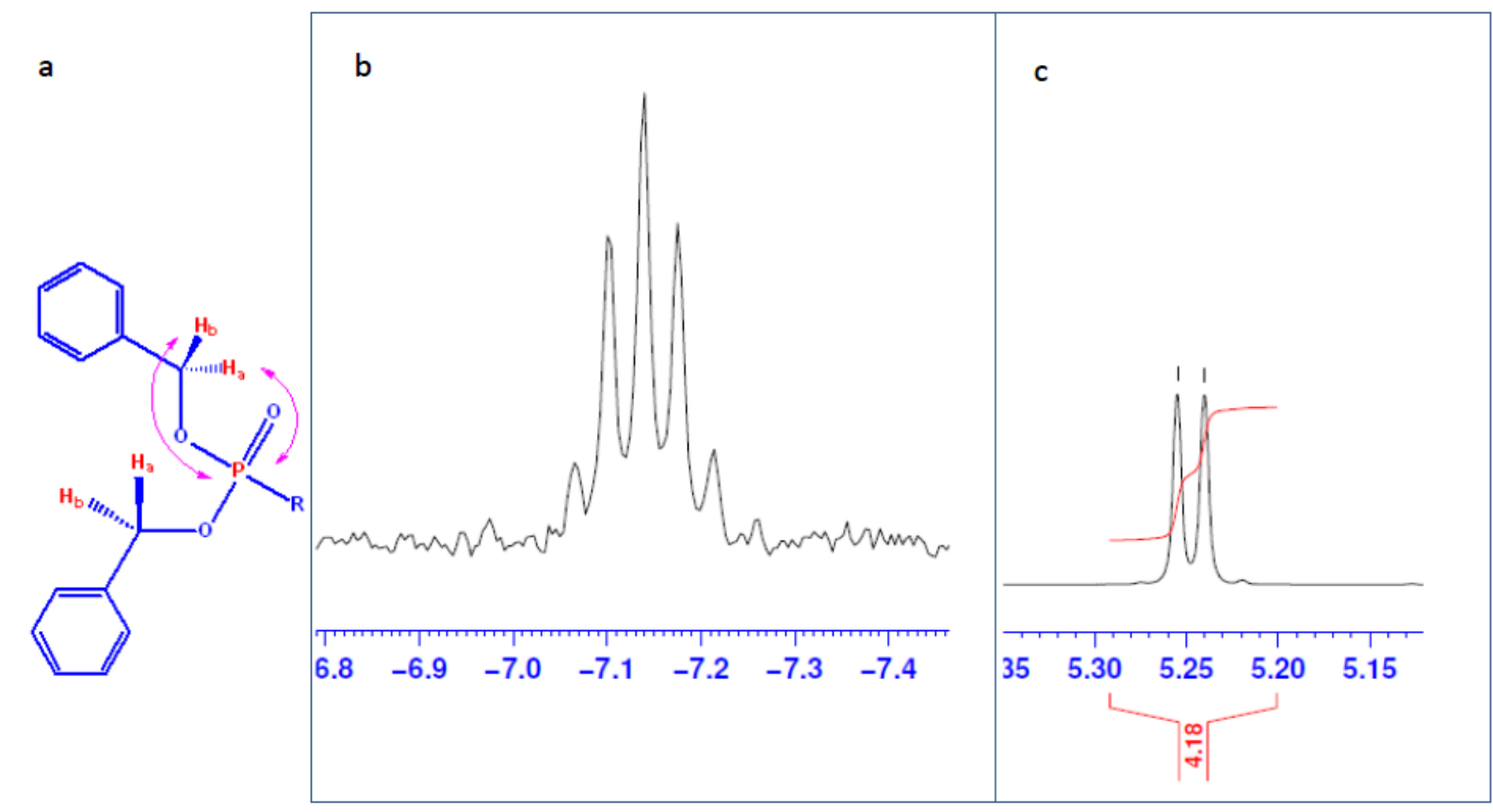

Figure 1: Shows representative NMR of dibenzyl phosphate signature splitting patterns (a) structure of dibenzyl protection of phosphorus center (b) Quintet splitting pattern of phosphorus in ${ }^{31} \mathrm{P}$ NMR (c) splitting pattern of $-\mathrm{CH}_{2}$ proton in ${ }^{1} \mathrm{H}$ NMR.

Studies ${ }^{27,}{ }^{28}$ have shown that the 5 position of Quercetin or any other flavonoids cannot be benzylated easily in the conditions stated in the synthesis since the 5-OH is less acidic than the rest of the phenolic moieties. This fact is attributed by an acid weakening effect of an intramolecular H-bond between the 5hydroxyl group and the 4-keto group. Therefore 4'- Quercetin monophosphate intermediate is preferred to 5- Quercetin monophosphate. The debenzylation of the intermediates was carried out by using TMSBr. The ${ }^{31} \mathrm{P}$ with proton decoupling confirmed the product with 4'-QP giving one peak and 5, 4'-QDP showing two peaks. 


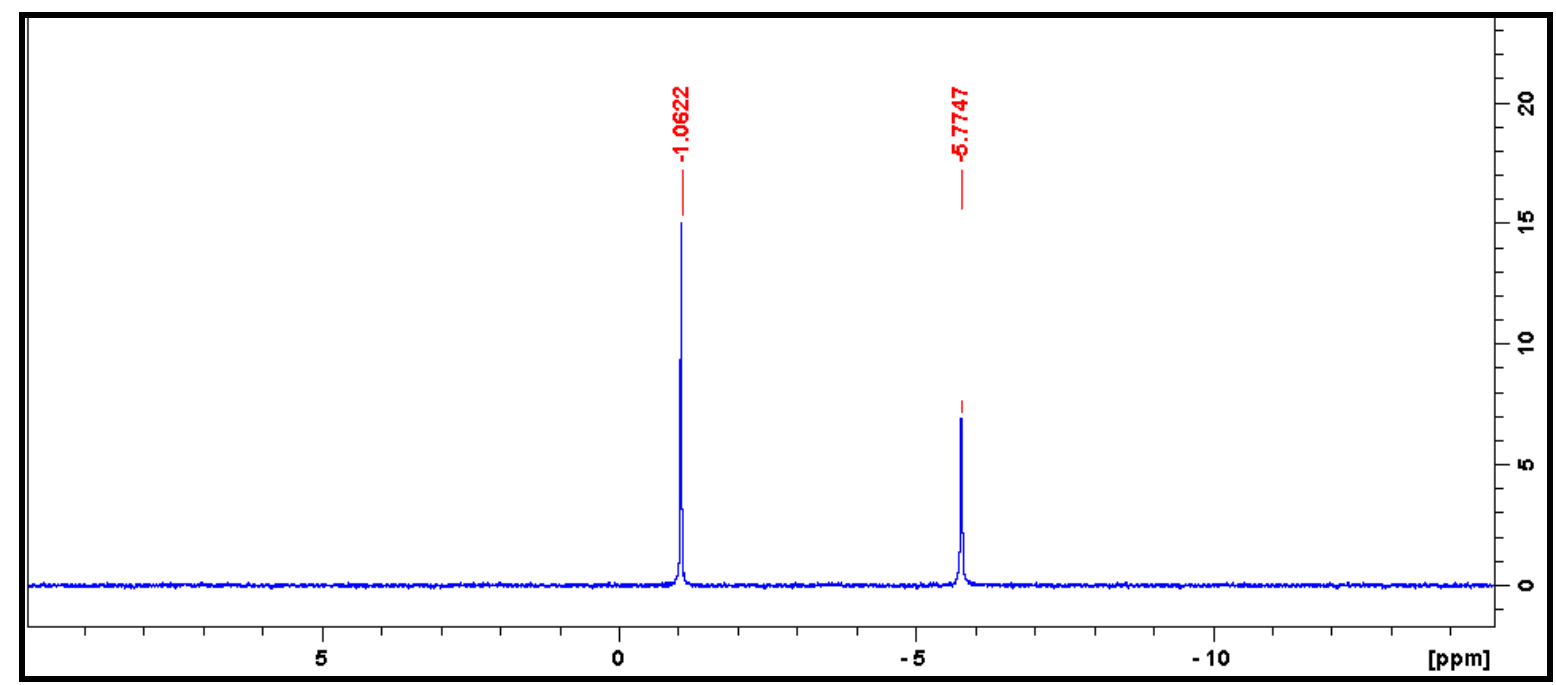

Figure 2: ${ }^{31} \mathrm{P}$ with proton decoupling of 5, 4'-Quercetin Diphosphate (QDP)

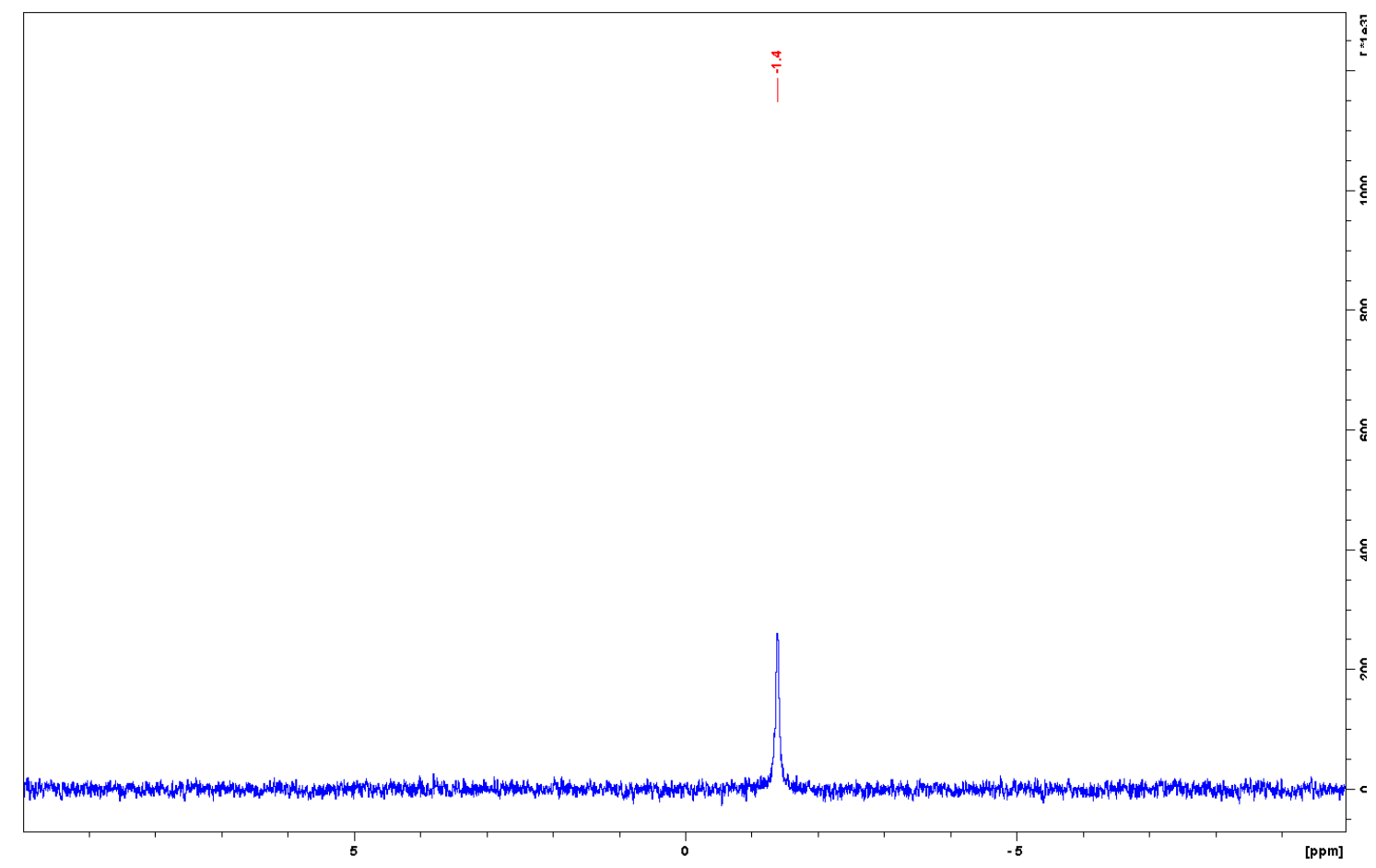

Figure 3: ${ }^{31} \mathrm{P}$ with Proton Decoupling of 4'-Quercetin Monophosphate (QP)

From reports ${ }^{29-31} 33,34$, we can reiterate that the more upfield -4.98 in QPPI is due to significant effect arising from electronegativity. The carbonyl group at position 4 which lies in conjugation with $\mathrm{C}-\mathrm{C}$ double bond at position 2 and 3 deprives the oxygen at position 3 electrons reducing the back-bonding ability which is consistent with Bent rule of electron withdrawing substituent requiring more p-character in the bonding orbital. ${ }^{37-38}$ Similarly, the enol ether oxygen at position 1 further complicates the electronegativity contribution and therefore the phosphorus at position 3 on ring $\mathrm{C}$ will be more upfield as seen in QPPI ${ }^{31} \mathrm{P}$ NMR. On the other hand, the splitting pattern of proton signals in the ${ }^{1} \mathrm{H}$ NMR resonating 
around 5ppm indicates the presence of the two methylene groups on the benzyl ether groups attached to phosphorus center. Ordinarily, these germinal protons are not expected to have any splitting since there is no vicinal proton neighbor or stereochemistry in the molecule, but due to the presence of the phosphorus nucleus at three bonds away, signals a powerful internuclear interaction as depicted in Figure 1.

However, the pattern in the ${ }^{1} \mathrm{H}$ spectrum quickly degenerate into complexity with increase in the number of the benzyl protected phosphates groups as seen in QPPI. This comes from superimposition of peaks and possible remote interaction of neighboring groups. We were therefore able to account for 20 methylene protons and 55 aromatic protons for QPPI (2) by integration corresponding to the claimed structure. Debenzylation of QPPI (2) yielded QPP (3) in 56\% yield.

In summary, we have successfully synthesized four intermediates 2, 8, 9 en-routes to Quercetin phosphate analogs with a yield of 47\%, $59.84 \%$ and $19.65 \%$ respectively from a reaction starting from Quercetin to give final products of compounds 3, 9 and 10 giving a yield of 56\%, 60.5\% and $85 \%$ respectively. On the other hand Apigenin triphosphate intermediate (ATRPI 14) and Apigenin Triphosphate (ATRP 15) gave a yield of $56.5 \%$ and $99 \%$ respectively.

\section{Acknowledgments}

The authors acknowledge the National Science Foundation CBET 1230189 for funding. The Regional NMR facility (600 $\mathrm{MHz}$ instrument) at SUNY-Binghamton is supported by NSF (CHE-0922815).

\section{References and notes}

1. Viskupicova, J.; Ondrejovic, M.; Maliar, T., Enzyme-Mediated Preparation of Flavonoid Esters and Their Applications. INTECH Open Access Publisher, 2012.

2. Rice-Evans, C. A.; Miller, N. J.; Paganga, G., Structureantioxidant activity relationships of flavonoids and phenolic acids. Free Radical Biology and Medicine 1996, 20 (7), 933-956

3. Wei,Y.,Peng,A.,Wang, B.,Ma, L.,Peng,G.,Du,Y., Tang,J.,Synthesis and biological evaluation of phosphorylated flavonoids as potent and selective inhibitors of cholesterol esterase. European Journal of Medicinal Chemistry 2014,74, 751758 .

4. Gao, Q.; Lian, G.; Lin, F., The first total synthesis of 7-<i $>$ $\mathrm{O}</ \mathrm{i}>-\beta$-d-glucopyranosyl-4'-<i $>\mathrm{O}</ \mathrm{i}>-\alpha-1-$-rhamnopyranosyl apigenin via a hexanoyl ester-based protection strategy. Carbohydrate research 2009, 344 (4), 511-515.

5. Williamson, G.; Manach, C. Bioavailabilty and bioefficacy of polyphenols in humans.II. Review of 93 intervention studies. Am. J. Clin. Nutr. 2005, 81 (Suppl.1), 243S-255S.
6. Biasutto, L.; Marotta,E.; Mattarei, A.; Beltramello, S.; Caliceti, P.; Salmaso, S.; Bernkop-Schnörch, A.;Garbisa,S.; Zoratti, M.; Paradisi, C. Absorption and metabolism of resveratrol carboxyesters and methane- sulfonate by explanted rat intestinal segments. Cell. Physiol. Biochem. 2009, 24, 557- 566.

7. Metodiewa, D.; Jaiswal, A.K.; Cenas, N.; Dickancaite', E. Quercetin may act as a cytotoxic prooxidant after its metabolic activation to semiquinone and quinoidal product. Free Radic. Biol. Med. 1999, 26, 107-116.

8. Chebil, L.; Humeau, C.; Falcimaigne, A.; Engasser, J.-M.; Ghoul, M., Enzymatic acylation of flavonoids. Process Biochemistry 2006, 41 (11), 2237-2251Beaucage, S. L.; Iyer, R. P., The synthesis of specific ribonucleotides and unrelated phosphorylated biomolecules by the phosphoramidite method. Tetrahedron 1993, 49 (46), 10441-10488.

9. Kim,M.K.,Park,K.,Lee,C.,Park,H, Choo,H.,Chong,Y., Enhanced Stability and Intracellular Accumulation of Quercetin by Protection of the Chemically or Metabolically Susceptible Hydroxyl Groups with a Pivaloxymethyl (POM) promoiety. J. M. Chem., 2010, 53, 8597-8607.

10. Tommasini, S.; Raneri, D.; Ficarra, R.; Calabrò, M. L.; Stancanelli, R.; Ficarra, P., Improvement in solubility and dissolution rate of flavonoids by complexation with $\beta$ cyclodextrin. Journal of Pharmaceutical and Biomedical Analysis 2004, 35 (2), 379-387.

11. Bouktaib, M.; Lebrun, S.; Atmani, A.; Rolando, C.Hemisynthesis of all the O- monomethylated analogues of Quercetin including the major metabolites, through selective protection of phenolic functions. Tetrahedron. 2002, 58, 10001-10009.

12. Zhou, A. .; Kikandi, S.; Sadik, O. A., Electrochemical degradation of quercetin: Isolation and structural elucidation of the degradation products. Electrochemistry Communications 2007, 9, 2246-2255. 
13. Kikandi S.N.; Musah, S.; Lee, K.; Hassani, J.;Shawn Rajan, S.; Zhou, A.; Omowunmi. A. Sadik, Comparative Studies of Quercetin Interactions with Monophosphate Nucleotides Using UV-Vis Spectroscopy and Electrochemical Techniques, Electroanalysis, 2007, 19(19-20), 2131-2140.

14. Zhou, A.; Sadik, O. A., Comparative Analysis of Quercetin Oxidation by Electrochemical, Enzymatic, Autoxidation, and Free Radical Generation Techniques: A Mechanistic Study. Journal of Agricultural and Food Chemistry 2008, 56, (24), 12081-12091.

15. Silverberg, L. J.; Dillon, J. L.; Vemishetti, P., A simple, rapid and efficient protocol for the selective phosphorylation of phenols with dibenzyl phosphite. Tetrahedron letters 1996, 37 (6), 771-774.

16. Atherton, F. R.; Todd, A. R., 129. Studies on phosphorylation. Part III. Further observations on the reaction of phosphites with polyhalogen compounds in presence of bases and its application to the phosphorylation of alcohols. Journal of the Chemical Society (Resumed) 1947, (0), 674-678.

17. Liu, D-k.; Liu, M.; Huang, C-j., Wang, Ping-bao .Preparation of dibenzyl phosphate, J. Chemical Reagents, 2005, 11 .

18. Okello, V. A.; Mwilu, S.; Noah, N.; Zhou, A.; Chong, J.; Knipfing, M. T.; Doetschman, D.; Sadik, O. A., Reduction of hexavalent chromium using naturally-derived flavonoids. Environmental Science \& Technology 2012, 46 (19), 1074310751.

19. Tanpure, R. P.; Nguyen, B. L.; Strecker, T. E.; Aguirre, S.; Sharma, S.; Chaplin, D. J.; Siim, B. G.; Hamel, E.; Lippert III, J. W.; Pettit, G. R., Regioselective synthesis of water-soluble monophosphate derivatives of combretastatin A-1. Journal of natural products 2011, 74 (7), 1568-1574.

20. Okello, V. A.; Osonga, F. J.; Knipfing, M. T.; Bushlyar, V.; \& Sadik, O. A. Reactivity, characterization of reaction products and immobilization of lead in water and sediments using quercetin pentaphosphate. Environ. Sci.: Processes Impacts, 2016, 18(3), 306-313

21. Pope, C. N. Organophosphorus Pesticides: Do they all have the same mechanism of toxicity? Journal of Toxicology and Environmental Health, Part B 1999, 2 (2), 161-181.

22. Potashman, M. H.; Duggan, M. E., Covalent modifiers: an orthogonal approach to drug design. Journal of medicinal chemistry 2009, 52 (5), 1231-1246.

23. Haché, B.; Brett, L.; Shakya, S., Phosphonate-free phosphorylation of alcohols using bis-(tert-butyl) phosphoramidite with imidazole $\cdot$ hydrochloride and imidazole as the activator. Tetrahedron Letters 2011, 52 (28), 3625-3629.

24. Beaucage, S. L.; Iyer, R. P., The synthesis of specific ribonucleotides and unrelated phosphorylated biomolecules by the phosphoramidite method. Tetrahedron 1993, 49 (46), 1044110488.

25. Fenton, O. S.; Allen, E. E.; Pedretty, K. P.; Till, S. D.; Todaro, J. E.; Sculimbrene, B. R., Catalytic Lewis acid phosphorylation with pyrophosphates. Tetrahedron 2012, 68 (44), 9023-9028.

26. Kargin, Y. M.; Budnikova, Y. H.; Martynov, B. I.; Turygin, V. V.; Tomilov, A. P., Electrochemical synthesis of organophosphorus compounds with $\mathrm{P}-\mathrm{O}, \mathrm{P}-\mathrm{N}$ and $\mathrm{P}-\mathrm{C}$ bonds from white phosphorus. Journal of Electroanalytical Chemistry 2001, 507 (1-2), 157-169.

27. Picq, M.; Prigent, A. F.; Chabannes, B.; Pacheco, H.Tetrahedron Lett. 1984, 25, 2227-2230

28. Slabbert, N. P. Tetrahedron 1977, 33, 821-824.

29. Koo, I. S.; Ali, D.; Yang, K.; Park, Y.; Wardlaw, D. M.; Buncel, E., Theoretical study of 31P NMR chemical shifts for organophosphorus esters, their anions and $\mathrm{O}, \mathrm{O}$ dimethylthiophosphorate anion with metal complexes. Bull Korean Chem Soc 2008, 29, 2252-8.

30. Dunn, E.; Purdon, J.; Bannard, R.; Albright, K.; Buncel, E., Correlation of 31P nuclear magnetic resonance chemical shifts in aryl phosphinates with Hammett substituent constants: Inductive versus resonance interactions and relevance to $\mathrm{p} \pi$ - $\mathrm{d} \pi$ bonding. Canadian journal of chemistry 1988, 66 (12), 3137-3142

31. Dominguez, Z.; Hernández, J.; Silva-Gutiérrez, L.; Salas-Reyes, M.; Sánchez, M.; Merino, G., Substituent Effects on 31P NMR Chemical Shifts and 1JP-Se of triarylselenophosphates. Phosphorus, Sulfur, and Silicon 2010, 185 (4), 772-784.

32. Smith, S. J.; Zimmer, H.; Fluck, E.; Fischer, P., Substituent Effects On The ${ }^{31} \mathrm{P},{ }^{13} \mathrm{C}$, and ${ }^{1} \mathrm{H}$ NMR Chemical Shifts Of Substituted Diphenyl 1-Phenylamino-1Phenylmethanephosphonates and their Anions. Phosphorous and Sulfur and the Related Elements 1988, 35 (1-2), 105-119.

33. Pedrosa, M. S.; Cajaiba Da Silva, J. F., AB-INITIO CALCULATIONS OF 31P NMR CHEMICAL SHIFTS OF
SUBSTITUTED ARYL DIALKYL PHOSPHATES. Phosphorus, Sulfur, and Silicon and the Related Elements 2001, 170 (1), 233-246.

34. Gorenstein, D. G., Dependence of phosphorus-31 chemical shifts on oxygen-phosphorus-oxygen bond angles in phosphate esters. Journal of the American Chemical Society 1975, 97 (4), 898-900.

35. Fu, L.; Jiang, Z.; cai, Z.; Liu, X.; He, H.; Yang, Y., Water-soluble phosphate prodrugs of pleuromutilin analogues with potent in vivo antibacterial activity against Gram-positive pathogens. Bioorganic \& Medicinal Chemistry Letters 2009, 19 (18), 5407-5410.

36. Alewood, P.; Perich, J.; Johns, R., Preparation of Na-(tButoxycarbonyl)-O-(dibenzylphosphono)-L-serine. Australian journal of chemistry 1984, 37 (2), 429-433.

37. Bent, H. A., An Appraisal of Valence-bond Structures and Hybridization in Compounds of the First-row elements. Chemical Reviews 1961, 61 (3), 275-311.

38. Gliga, A.; Goldfuss, B.; Neudörfl, J. M., Lithium phosphonate umpolung catalysts: Do fluoro substituents increase the catalytic activity? Beilstein journal of organic chemistry 2011, 7 (1), 1189 1197.

39. Representative Spectroscopic data : Compound 7 1H NMR (600 MHz, DMSO-d6): d 7.90 (s, 1H), $7.86(\mathrm{~d}, 1 \mathrm{H}, \mathrm{J}=8.7), 7.85(\mathrm{~d}$, $1 \mathrm{H}, \mathrm{J}=8.6 \mathrm{~Hz}), 7.50-6.85(\mathrm{~m}, 40 \mathrm{H}), 5.33(\mathrm{q}, 4 \mathrm{H}, \mathrm{J}=9.12 \mathrm{~Hz})$, $5.32(\mathrm{~d}, 4 \mathrm{H}, \mathrm{J}=9.0 \mathrm{~Hz}), 5.26(\mathrm{q}, 4 \mathrm{H}, \mathrm{J}=7.3 \mathrm{~Hz}) ; 13 \mathrm{C}$ NMR $(600$ $\mathrm{MHz}$, DMSO-d6): 178.2,164.2,161.0,156.2,155.5,136.9,136.6,136.3,136.1,128.7,128. $5,128.4,128.1,128.0,127.9,127.5,125.3,122.1,114.1,105.4$, 98.50, 93.23, 73.44, 70. 02, 69.7, 69.4: 31P NMR (600 MHz, DMSO-d6): $-5.86(\mathrm{JP}-\mathrm{H}=19 \mathrm{~Hz}),-7.27(\mathrm{JP}-\mathrm{H}=21 \mathrm{~Hz})$.

;Compound $14{ }^{1} \mathrm{H}$ NMR (600 MHz, DMSO-d $\left.{ }_{6}\right)$ : d 8.07 (d, 2H, J = $8.4), 7.41-7.28(\mathrm{~m}, 33 \mathrm{H}), 7.12(\mathrm{~s}, 1 \mathrm{H}), 6.93(\mathrm{~s}, 1 \mathrm{H}), 5.22(\mathrm{t}, 8 \mathrm{H}, \mathrm{J}$ $=8.2 \mathrm{~Hz}), 5.29(\mathrm{t}, 4 \mathrm{H}, \mathrm{J}=6.3 \mathrm{~Hz}) ;{ }^{31} \mathrm{P}$ NMR $(600 \mathrm{MHz}$, DMSO$\left.\mathrm{d}_{6}\right):-6.70\left(\mathrm{~J}_{\mathrm{P}-\mathrm{H}}=18 \mathrm{~Hz}\right),-7.09\left(\mathrm{~J}_{\mathrm{P}-\mathrm{H}}=18 \mathrm{~Hz}\right),-7.52 \mathrm{~J}_{\mathrm{P}-\mathrm{H}}=19$ $\mathrm{Hz}$ ).Figure S2E gives ${ }^{13} \mathrm{C}$ NMR data for the compound.

\section{Supplementary Material}

Additional information on the structural elucidation of the compounds such as ${ }^{1} \mathrm{H},{ }^{13} \mathrm{C},{ }^{31} \mathrm{P}$ NMR. This material is available free of charge via Internet at http://dx.doi.org/.... 


\section{GRAPHICAL ABSTRACT}

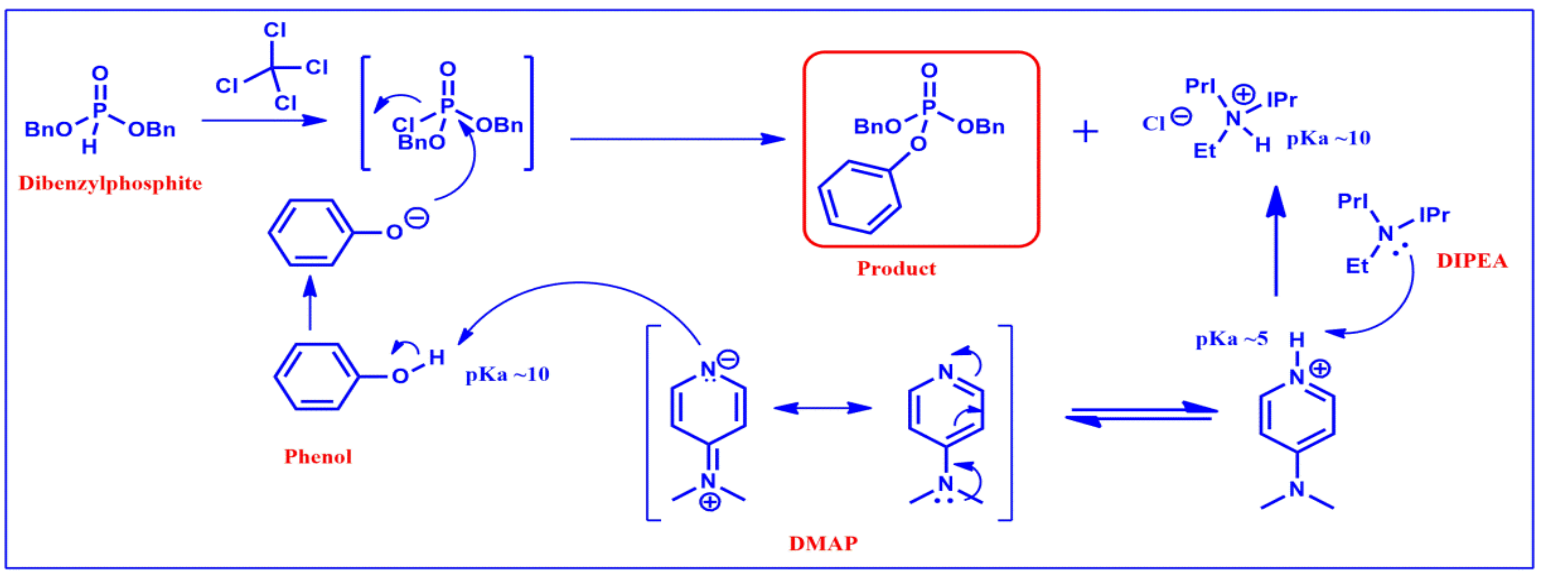

